\title{
Mögliche wissenschaftliche Ansätze als Erklärungsgrundlage komplementärmedizinischer Therapien
}

\author{
Urs Weilenmann
}

Zürich, Schweiz

Schlüsselwörter

Ganzheitsmedizin - Chaostheorie - Quantenphysik ·

Wasser · Biophotonen · Impfkomplikation

\section{Zusammenfassung}

Im Zusammenhang mit komplementärmedizinischen Therapien wird immer wieder der Begriff unwissenschaftlich verwendet. In dieser Arbeit werden verschiedene physikalische Theorien, die eine mögliche Basis für die beobachteten Phänomene bieten, kurz vorgestellt. Gemeinsam ist ihnen, dass sie Erkenntnisse aus der Chaos- und Quantentheorie auf Organismen anwenden. Demnach können beispielsweise kleinste Reize, wie sie bei gewissen ganzheitlichen Therapien auftreten, als Resonanzphänomene von oszillierenden Systemen erkannt werden. Als Folge erscheint das übliche, auf klassischen Ansätzen basierende biochemische Denkmodell als unvollständig, wie anhand einer Impfkomplikation gezeigt wird.

In der Diskussion um komplementärmedizinische Verfahren wird von den Skeptikern immer wieder darauf hingewiesen, dass deren wissenschaftliche Basis nicht fundiert ist. Dies widerspricht unzähligen Erfolgsberichten in entsprechenden Fachzeitungen und auch meiner eigenen Praxiserfahrung $[1,2]$. Zusätzlich ist die Frage nach der Placebowirkung sicher gerechtfertigt; ebenso muss die Frage nach dem Geltungsbereich der jeweiligen Überlegungen gestellt werden, mit denen diese Berichte als unwissenschaftlich betitelt werden. Im Folgenden werden einige Theorien vorgestellt, die mögliche Erklärungsansätze für komplementärmedizinische Phänomene bieten. Ausserdem wird hinterfragt, ob man mit klassischen naturwissenschaftlichen Methoden solche Therapien widerlegen kann.

\author{
Keywords \\ Holistic medicine - Chaos theory - Quantum physics . \\ Water $\cdot$ Biophotons Vaccination damage
}

\begin{abstract}
Summary
Possible Scientific Approaches as an Explanatory Basis for Therapies of Complementary Medicine

In context with therapies of complementary and holistic medicine, the term non-scientific is often used. In this article, various scientific theories, which may form a basis for the described phenomena, are briefly introduced. They all have in common that they apply the knowledge gained from chaos and quantum theory to organisms. For instance, extremely small impulses, as they occur in certain complementary therapies, can therefore be identified as resonance phenomena of oscillating systems. As a consequence, the common biochemical way of thinking seems to be incomplete, as shown by the example of a vaccination damage.
\end{abstract}

Gemäss Brockhaus Enzyklopädie bedeutet wissenschaftliche Methodik ein gesichertes, in einen Begründungszusammenhang von Sätzen gestelltes und damit intersubjektiv kommunizierbares und nachprüfbares Wissen. Die Methoden richten sich dabei nach dem zu untersuchenden Gegenstand. Dabei haben sich zwei getrennte Wissenstraditionen herausgebildet: Die Naturwissenschaften, die aufgrund von Beobachtungen, Hypothesen, Theorien und reproduzierbaren Experimenten arbeiten, und die geisteswissenschaftliche Methode (Hermeneutik), die auf niemals gänzlich zu erschöpfenden Phänomenen und in der Beobachtung einholbarer Deutung individueller Phänomene, auf Bedeutungszuweisung und Sinnverstehen beruht. 
Diese Definition geht letztlich auf René Descartes zurück. Für ihn bestand die Natur, neben Gott, aus zwei ganz voneinander getrennten Substanzen. Es gab das reine Denken ohne jede Räumlichkeit (res cogitans) und die reine Ausdehnung ohne jedes Denken (res extensa), die einer genauen mathematischen Beschreibung zugänglich ist [3].

Lebewesen zeichnen sich durch eine ganze Anzahl besonderer Eigenschaften aus, wie z.B. hochkomplexe Moleküle (DNA usw.), Wachstum, Tod, Reproduzierbarkeit, Anpassung an Umwelteinflüsse und - im Fall des Menschen - Bewusstsein. In den Naturwissenschaften wird das Phänomen «Leben» ausschliesslich mit Methoden beschrieben, die sich bei unbelebter Materie bewährt haben. Welche Methode nun die geeignetste ist, um einen Organismus zu beschreiben, ist jedoch ungeklärt. Es fällt auch auf, dass in der Schulmedizin hauptsächlich mit Erkenntnissen der klassischen Physik argumentiert wird. Ob dies genügt, ist wissenschaftlich nicht geklärt.

Von verschiedener Seite wird seit Jahren darauf hingewiesen, dass Erkenntnisse aus der Chaostheorie und der Quantenphysik grundlegend für das Verständnis von Lebewesen sind [4]. Falls dem so ist, bedeutet das für die Biologie, dass die ausschliesslich klassische Beschreibung eines Lebewesens grundsätzlich überdacht werden muss. Umso mehr trifft das auch auf die Beurteilung von ganzheitlichen Therapien wie Homöopathie, Akupunktur, Bioresonanz und anderen zu.

Folgende Argumente deuten darauf hin, dass die allein auf klassischen physikalischen Methoden basierende Beschreibung unvollständig ist:

Ilya Prigogine (Nobelpreis 1977) kritisierte den Ansatz der klassischen Physik: «Den Vorstellungen der klassischen Physik lag die Überzeugung zugrunde, dass die Zukunft durch die Gegenwart determiniert sei und man daher durch ein sorgfältiges Studium der Gegenwart die Zukunft enthüllen könne. Das war natürlich nie mehr als eine theoretische Möglichkeit. Man könnte sie vielleicht als den grundlegenden Mythos der klassischen Wissenschaft bezeichnen» [4].

Prigogine entwickelte die Theorie dissipativer Strukturen, indem er zeigen konnte, dass chemische Reaktionen unter Energieverbrauch und weit weg vom thermischen Gleichgewicht Ordnungsstrukturen entwickeln können. In seinem Nobel-Vortrag sagte er: «Weit weg vom Gleichgewicht kommt demnach eine unerwartete Beziehung zwischen der chemischen Kinetik und der Raum-ZeitStruktur von reagierenden Systemen zum Vorschein» [4].

Später wies er darauf hin, dass Lebewesen die Welt mit einem Zeitpfeil, also nicht reversibel, erleben. Die klassische Physik hat gemäss Prigogine auf paradoxe Weise diesen Zeitpfeil geleugnet. Er zeigt, dass mit der Entwicklung der Physik dissipativer Systeme dieses Paradoxon überwunden werden kann, wobei Ordnungsstrukturen stets aus vorangegangenen Instabilitäten hervorgehen.
Nun sind die meisten interessanten Systeme, von der Mechanik bis zur Quantenmechanik, solche instabilen Systeme. In ihnen können sich kleinste Störungen mit der Zeit verstärken und das Systemverhalten beeinflussen [5]. Diese Erkenntnisse sind grundlegend für das Verständnis von Lebewesen. Als eine Konsequenz folgt daraus, dass Untersuchungen nicht immer reproduzierbar sind.

Für den Mathematiker Roger Penrose kann eine «wissenschaftliche Weltauffassung wohl kaum ernsthaft den Anspruch erheben, abgeschlossen und vollständig zu sein, wenn sie das Problem des Bewusstseins unerklärt lässt. Bewusstsein ist ein Teil unserer Welt, und eine physikalische Theorie, die ihm keinen Platz einräumt, kann keine angemessene Beschreibung der Welt darstellen» [6]. Als profunder Kenner der Quantenphysik zieht er diese als Möglichkeit in Betracht, möchte sie aber in Bezug auf die Gravitation erweitern.

Kurt Gödel hat mit seinem Unvollständigkeitssatz gezeigt, dass kein mathematisches System seine eigene Widerspruchsfreiheit beweisen kann. Das bedeutet, dass sich Verständnis und Einsicht nicht auf ein System von Rechenregeln oder Statistiken reduzieren lassen. Gemäss Penrose ist dieser Unentscheidbarkeitssatz der vielleicht grundlegendste, der je in der Mathematik geleistet wurde [6]. Der Chemiker Friedrich Cramer meint dazu, Gödels Entdeckung bedeute die Unmöglichkeit von prinzipiellen Lösungen, den Abschied vom Prinzipiellen an sich [4]. Damit beginnt sich abzuzeichnen, dass das Reduzieren von medizinischer Forschung auf Doppelblindstudien oder reproduzierbare Einzeldarstellungen aus dieser Sicht falsch ist.

Thomas Görnitz - als ein Physiker, der sich mit dieser Thematik befasst hat - beschreibt in seinem Buch «Quanten sind anders», dass das eigentliche Wesen dieser neuen Physik die Beziehung von Teilchen untereinander und zum Ganzen ist. Der Physiker Carl Friedrich von Weizsäcker sagt hierzu, dass es einen grossen Ansatz zur längst fälligen Interpretation der Quantenphysik darstellt. Görnitz stellt dieses Konzept der herkömmlichen Beschreibung gegenüber, die er als eine Physik der Objekte darstellt. Vereinfacht kann man sich vorstellen, dass sich im klassischen Fall die Beziehungen von Teilchen eher additiv zueinander verhalten, während im Quantenfall eine reichhaltigere, multiplikative Beziehung besteht.

Dabei können neue Eigenschaften entstehen. Er schrieb: «Der holistische Charakter der Quantentheorie ist ihr wesentlichstes Merkmal und bildet den Hauptunterschied zum klassischen Fall» [7]. Holismus liegt vor, wenn das Ganze mehr ist als die Summe seiner Teile. Für mich ist diese Bedingung bei einem System mit Bewusstsein erfüllt. Das ausschliessliche Festhalten am klassischen Modell in grossen Kreisen der Biowissenschaften und der Medizin kann Görnitz nur aus historischer Sicht verstehen. 
Im Folgenden werden einige Beobachtungen besprochen, die auf Phänomenen der Chaostheorie und der Quantenphysik beruhen (Tab. 1) und an einem Patientenbeispiel eine mögliche Konsequenz erläutern.

\section{Elektrische Eigenschaften von biologischen Systemen}

Als einer der Ersten erkannte wohl Fröhlich, Professor für theoretische Physik in Liverpool, die mögliche Bedeutung der erstaunlichen elektrischen Eigenschaften von Zellen. Eine Potenzialdifferenz von gegen $100 \mathrm{mV}$ über eine Zellwand von $10 \mathrm{~nm}$ entspricht einem enormen Feld von circa 10 Millionen Volt/Meter. Das dauernde Depolarisieren und die daraus folgenden elektromagnetischen Schwankungen müssen polare Moleküle zum Schwingen bringen. Fröhlich vermutete, dass diese oszillierenden Felder einander beeinflussen. Die Frequenzen berechnete er aus der Membrandicke und der Geschwindigkeit, mit der sich longitudinal polarisierte Wellen in Materie fortpflanzen. Es ergaben sich Resonanzfrequenzen von $10^{11}$ bis $10^{12} \mathrm{~Hz}$, was Wellenlängen im Mikrowellenbereich entspricht.

Durch seine Beschäftigung mit Supraleitung, einem Phänomen, bei dem einzelne Teile eines Systems plötzlich ihre Individualität verlieren und kollektiv als Einheit reagieren, vermutete er, dass ähnliche Eigenschaften bei polaren Molekülen einer Zellwand auftreten können. Das würde bedeuten, dass kollektives, kohärentes Verhalten, wie es in der unbelebten Natur nur bei speziellen Bedingungen, z.B. bei ganz tiefen Temperaturen als quantenphysikalisches Phänomen, vorkommt, in Lebewesen bei Zimmertemperatur auftreten kann.

Zahlreiche Experimente unter anderem aus Russland oder am Max-Planck-Institut für Festkörperphysik in Stuttgart zeigten spezifische Effekte an Zellen nach Bestrahlung mit den von Fröhlich berechneten Frequenzen $[8,9]$. Dies ist nur möglich, weil Organismen physikalisch offene, dissipative Systeme sind, die weit weg von thermischem Gleichgewicht existieren.
Wesentlich an Fröhlichs Modell dieser kohärenten elektromagnetischen Kopplung ist, dass es die Aufmerksamkeit von biologischen Strukturen auf biologische Funktionalität lenkt. So wies John Swain (Northeastern University Boston, USA, und CERN, Genf, Schweiz) auf die Möglichkeit hin, dass die Fröhlich-Modi sich über das gesamte elektromagnetische Spektrum erstrecken und damit die Basis für elektromagnetische intrazelluläre und interzelluläre Kommunikation bilden könnten [10].

Hyland [9] unterstreicht weiterhin, dass Fröhlichs Arbeiten die Grundlage zum Verständnis bieten, dass unter gewissen Bedingungen ganz schwache elektromagnetische Strahlungen von spezifischen Frequenzen ein System beeinflussen können. Zusätzlich stehen sie auch im Einklang mit den Theorien, dass makroskopische Quanteneffekte, wie sie im Zusammenhang mit Bewusstseinsphänomenen diskutiert werden, vorkommen können [11].

Auf mögliche Auswirkungen von Fröhlichs Theorien zum Krebsproblem weist Pokorný [12] hin. Er erwähnt, dass die beschriebenen kohärenten elektromagnetischen Felder eine grundlegende Bedeutung für die Organisation, den Transport und die Kommunikation von Zellbestandteilen haben. Eine wichtige Rolle spielen in dem Zusammenhang die Mikrotubuli als Hauptorganisatoren des Zytoskeletts und ihnen angelagerte Mitochondrien, in denen die Energieproduktion abläuft. Einerseits konnte man zeigen, dass an der Aussenmembran der Mitochondrien sehr grosse elektrische Felder von bis 3,5 Millionen Volt/Meter entstehen (Abb. 1), die wohl die meisten Moleküle in der Umgebung beeinflussen. Andererseits weist er darauf hin, dass bei der Zellatmung nur etwa $40 \%$ der Energie in Form von ATP (Adenosintriphosphat) gespeichert wird. Der Rest, oft als «wasted energy» bezeichnet, wird als Wärme und Ultraviolettstrahlung abgegeben. Er zeigt nun, dass diese Energie gebraucht wird, um mechanische Vibrationen an den Mikrotubuli zu erzeugen. Diese Umwandlung von Energie in mechanische Schwingungen ist für Pokorný eine fundamentale Eigenschaft von biologischen Systemen, wobei die Mikrotubuli die Haupt-

Tab. 1. Subjektive und unvollständige Zusammenstellung von Eigenschaften der drei grossen naturwissenschaftlichen Theorien

\begin{tabular}{lll}
\hline Klassische Physik & Chaostheorie & Quantenphysik \\
\hline Entspricht der Alltagslogik & Unbestimmtheit in Makrosystemen & «Unlogisch» in unserem Alltagsverständnis \\
Deterministisch & Zeitpfeil & Welle-Teilchen-Dualismus, \\
& Soppelcharakter der Materie \\
Kausalitätsprinzip & $\begin{array}{l}\text { Hochsensibel auf Änderung } \\
\text { der Anfangsbedingungen }\end{array}$ & $\begin{array}{l}\text { Superpositionsprinzip } \\
\text { Reproduzierbar }\end{array}$ \\
Apriori-Begriffe von Raum und Zeit & Nur bedingt reproduzierbar & Unbestimmtheit im Mikrosystem \\
& & \\
Physik der Objekte & Fraktale Dimension/Geometrie & Verschiedenste Interpretationen möglich, \\
\end{tabular}






Abb. 1. Rund um die Mitochondrien wurde mithilfe von fluoreszierenden Partikeln (schwarze Punkte) ein elektrostatisches Feld von bis zu 3,5 Millionen Volt/Meter nachgewiesen. Dessen Richtung wird durch die Pfeile angegeben. Energie, die nicht für die ATP-Produktion in den Mitochondrien benötigt wird, wird in Form von Wärme und UV-Strahlung in dieses Feld abgegeben. Dabei entstehen mechanische Schwingungen, wobei die Mikrotubuli als Hauptbestandteile des Zytoskeletts eine wichtige Rolle spielen [12].

strukturen von Fröhlichs kohärenten Vibrationen zu sein scheinen. Es wird nun vermutet, dass Dysfunktionen von Mitochondrien, wie man sie bei Krebszellen findet, mit Störungen dieses komplexen Systems aus mechanischen und elektromagnetischen Schwingungen zusammenhängen.

\section{Die mögliche Rolle des Wassers in lebenden Organismen}

Wasser hat im Gegensatz zu anderen Flüssigkeiten verschiedene Anormalitäten, wie etwa die anormale Temperaturabhängigkeit der Dichte, die anormale Druckabhängigkeit der Viskosität usw. Insgesamt sind es über 60 Anormalitäten, die man anhand der Struktur des Wassers erklären muss. Trincher [13] wies beispielsweise darauf hin, dass Wasser im Temperaturbereich von 0 bis $60^{\circ} \mathrm{C}$ eine aus drei verschiedenen Phasen zusammengesetzte Flüssigkeit ist. Am wichtigsten sind für uns die echte flüssige Phase und eine quasikristalline, die man sich wie aus noch nicht ganz geschmolzenen Eiskristallen vorstellen kann, die im Wasser gelöst sind. Interessant ist, dass Wasser gerade um $37^{\circ} \mathrm{C}$ das Minimum der spezifischen Wärme aufweist und daher ein Maximum an Strukturmöglichkeiten zeigt. Das ist deshalb der Fall, weil das Verhältnis der flüssigen zur quasikristallinen Phase ausgeglichen ist. Da Lebewesen zum grössten Teil aus Wasser bestehen, fragt Trincher, ob es Zufall ist, dass die höchstentwickelten Organismen gerade in diesem Bereich ihre Körpertemperatur haben [13].

Der italienische Physiker Del Giudice beschäftigt sich, basierend auf der Quantenelektrodynamik (QED), mit der Theorie, dass die verschiedenen Anregungszustände von Atomen und Molekülen in Lebewesen mit einem fluktuierenden Vakuumfeld interagieren. Dadurch entstehen kleine Areale, in denen die beteiligten Moleküle in einem hoch geordneten Zustand vorkommen. Diese Areale werden als «Coherent Domains» (CD), kohärente Bereiche, bezeichnet. Sie haben beim Wasser einen Durchmesser von gegen $100 \mathrm{~nm}$, was einer Menge von etwa $10^{7}$ Molekülen entspricht. Die Lebensdauer solcher kohärenten Bereiche wird als sehr lange geschätzt, d.h. Monate oder länger. Umgeben sind sie von «normalem» Wasser (engl. bulk water). Auch hier ergeben sich aus der Struktur heraus verschiedene physikalische Eigenschaften. So stossen z.B. hoch geordnete, kohärent schwingende Moleküle weniger aneinander, was zu einer anderen Temperatur und Dichte führen muss. Ausserdem geben Wassermoleküle im kohärenten Zustand eher Elektronen ab. Wasser kann so in sich selbst wie eine Batterie wirken, wobei das nicht kohärente Wasser den positiven Pol darstellt.

Man hat zudem entdeckt, dass flüssiges Wasser entlang hydrophiler Oberflächen ähnliche Eigenschaften zeigen kann wie in den erwähnten kohärenten Bereichen. So bildet sich zuerst eine Schicht von hochgeordneten Wassermolekülen mit ganz anderen Eigenschaften als im dahinter liegenden ungeordneten, «normalen» Wasser. Diese Wasserschicht, die den erwähnten kohärenten Bereichen entspricht, wird als Exclusion Zone (EZ) Water oder auch als Interfacial (IF) Water bezeichnet [14].

Doch was bedeutet das? Der Biochemiker Voeikov und der Physiker Del Giudice fassen die für biologische Systeme wichtigsten Eigenschaften zusammen:

- EZ-Wasser bildet eine kohärente Struktur.

- Da die Moleküle viel kompakter zusammenliegen, können Fremdstoffe viel schlechter penetrieren.

- EZ-Wasser hat ein negatives elektrisches Potenzial zum angrenzenden normalen Wasser von bis $-150 \mathrm{mV}$.

- Die Dicke der EZ-Schicht nimmt zu, wenn sie mit sichtbarem Licht und speziell mit Infrarot bestrahlt wird.

Aufgrund der erwähnten Eigenschaften kommen Voeikov und Del Giudice zu dem Schluss, dass Wasser im EZ-Zustand Energie speichern kann. Doch als wichtigste Folgerung gilt, dass eine kohärente Wasserdomäne (CD) fähig ist, niederwertigere («low-grade») Energie mit grosser Entropie aus der Umgebung in hochwertige («high-grade») Energie mit tiefer Entropie zu transformieren. Dieses kohärente Wasser kann dann unter Abgabe von Photonen wieder in normales Wasser umgewandelt werden. Dazu wird Sauerstoff gebraucht, und es ergibt sich ein Prozess, der an eine Verbrennung erinnert $[15,16]$. Wasserverbrennung als Spezialität von Organismen!

Auf einen weiteren möglichen Effekt von geordneten Wasserstrukturen weist Penrose [6] hin. Ausgehend von Fröhlichs Theorie von quantenkohärentem Verhalten in Zellen sieht er in den Mikrotubuli mögliche morphologische Strukturen, in denen solche Phänomene entstehen können. Dabei spielt geordnetes Wasser, das hier als «vicinales» Wasser bezeichnet wird, als Isolator eine entscheidende Rolle $[6,11]$. 


\section{Biophotonen}

Lebewesen senden schwaches Licht aus. Aufgrund der gemessenen Frequenzen und der Intensität wird vermutet, dass Zellen dank dieser Photonenstrahlung miteinander kommunizieren können. Vermutlich können sie als Spezialfall von Fröhlichs Theorie aufgefasst werden [10]. Diese Biophotonenstrahlung wird definiert als von Zellen abgegebene ultraschwache Lichtemission (einzelne bis einige hundert Photonen pro $\mathrm{cm}^{2}$ und Sekunde), die ein Spektrum von 200 bis $800 \mathrm{~nm}$ umfasst und von einem kohärenten, delokalisierten elektromagnetischen Feld in Organismen produziert wird [17].

Ein eindrückliches Experiment gelang in dem Zusammenhang dem Biologen Galle [18]. Er konnte die Biophotonenstrahlung von Wasserflöhen (Daphnia magna) (Abb. 2) messen. Gibt man nun mehrere Wasserflöhe in eine Küvette, erwartet man, dass die Photonenstrahlung stetig ansteigt. Es zeigte sich aber eine ganz andere Kurve. Bei einer gewissen Anzahl von Tieren gab es plötzlich ein Absinken der Intensität, dann wieder eine Zunahme, ein Absinken usw. Mit anderen Worten bedeutet das, dass die Dichte der Zellen in der Küvette die Biophotonenstrahlung nicht linear beeinflusst. Popp [10] interpretiert, dass die Tiere mit ihren Photonenstrahlungen, etwa via positiver und negativer Interferenzen, miteinander kommunizieren. Das Experiment zeigt somit, dass interzelluläre Kommunikation durch elektromagnetische Strahlung existieren muss, und bestätigt damit ältere Theorien, wie sie vor allem von Alexander Gurwitsch in den 1920er-Jahren aufgestellt wurden. Unterschiede in der Biophotonenstrahlung von gesundem und Tumorgewebe deuten auf ähnliche Mechanismen hin.

Aus dieser Sicht sind die Biophotonen vermutlich das wichtigste Informationssystem eines Organismus: «This means that they are providing an essential, if not the most essential information channel in living systems» [19]. Andererseits verändert auch die Materie ständig das Biophotonenfeld, woraus sich eine dauernde gegenseitige Beeinflussung ergibt. Das hilft auch zu erklären, warum so viele biochemische Reaktionen, wie z.B. die DNAReplikation, mit einer so kleinen Fehlerrate ablaufen können. Für diesen Mechanismus bietet die klassische Biochemie meines Wissens keine genügenden Erklärungsansätze.

Theoretisch können diese Photonen auf verschiedene Weise abstrahlen. Entweder einfach zufällig, man spricht dann von chaotischer Abstrahlung, oder sie können einander beeinflussen. In diesem Fall spricht man von einem kohärenten Verhalten. Messungen lassen den Schluss zu, dass genau dieses Kohärenzverhalten grundlegend für Organismen ist und der Grad der Kohärenz viel über den Gesundheitszustand aussagen kann. Es gibt außerdem in der Quantenphysik einen Spezialfall, wie sich Photonen

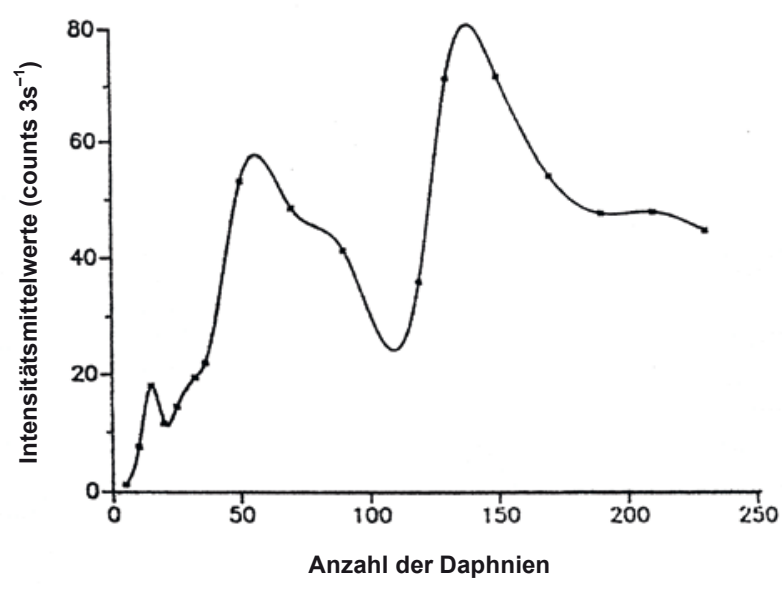

Abb. 2. Intensitätsmittelwerte (counts $3 \mathrm{~s}^{-1}, \mathrm{n}=4-6$ ) der Messpopulationen 1-6 als Funktion der Anzahl der Daphnien in 15 ml Volumen. Die Werte sind durch eine Ausgleichskurve (Spline) miteinander verbunden [18].

verhalten können, den man «gequetschtes Licht» (engl. squeezed light) nennt.

Ausgehend von der Unschärferelation von Heisenberg, die besagt, dass man von einem Teilchen nie genau den Ort und den Impuls beschreiben kann, gibt es eine ähnliche Eigenschaft bei Photonen. Man kann nie genau die Frequenz oder die Wellenlänge messen; es gibt eine gewisse Unschärfe. Dem internationalen Institut für Biophysik in Neuss, Deutschland, gelang es, solche gequetschten $\mathrm{Zu}-$ stände bei Biophotonen nachzuweisen [20]. Gequetschte Photonen verleihen dem Licht gemäß Popp nahezu ans Wunderbare grenzende Eigenschaften. Einerseits ermöglichen sie einem elektromagnetischen Feld, die Ortsunschärfe durch Frequenzüberlagerungen so zu verkleinern, dass fast beliebig kleine Regionen, sogar subatomare Strukturen, angesteuert werden können, und andererseits kann fast beliebig viel Information übermittelt werden. Es wäre daher ein ideales Steuerungselement für so ein komplexes Vielteilchensystem wie einen Organismus [10].

\section{Skalarwellen}

Als Tachyonen oder Skalarwellen bezeichnet man nichtelektromagnetische Felder, die man bis heute nicht direkt messen kann und die daher hypothetischer Natur sind. Sie bilden als sog. feinstoffliche Felder ein Bindeglied zwischen Physik und Esoterik. Experimentell hat wohl Nicola Tesla (1856-1943) als Erster bewusst solche Wellen erzeugt, weshalb oft auch von Teslawellen gesprochen wird. Erklärungen bot die damals diskutierte Äthertheorie, derzufolge das Vakuum nicht einfach leer ist, sondern von einer unbekannten Substanz, dem «Äther», durchdrungen wird.

Vereinfacht kann man sich bei Skalarwellen vorstellen, dass elektromagnetische Wellen nicht nur transversal 
schwingen, sondern immer auch einen longitudinalen Schwingungsanteil besitzen, ähnlich der Schallwellen [21].

Gemäss dem amerikanischen Kernphysiker Thomas E. Bearden können die Skalarwellen als eine Art Longitudinalwelle mit Fluktuationen im Vakuum interpretiert werden [22]. Für uns interessant sind seine Schlussfolgerungen bezüglich der Biologie:

- Skalarwellen sind fundamentaler als elektromagnetische Wellen.

- Sie durchdringen einen Faradayschen Käfig.

- Ihre Überlagerung erzeugt elektromagnetische Felder.

- Sie übertragen keine Energie, sondern Informationen.

- Sie stellen einen möglichen Bereich dar, in dem Materie und Bewusstsein aufeinander einwirken können.

Da Bearden das Vakuumfeld als Schnittstelle zwischen Materie und Bewusstsein ansieht, vertritt er die Ansicht, dass Skalarwellen das Bewusstsein beeinflussen wie auch die Psyche umgekehrt die Skalarwellen beeinflussen kann. Er ist auch überzeugt, dass Popps Biophotonenstrahlung eine Seite eines fundamentaleren Skalarwellenprozesses ist [22].

In den letzten Jahren wurden in der Komplementärmedizin einige Geräte entwickelt, die gemäss Herstellerangaben dazu konstruiert sind, künstlich erzeugte Skalarwellen zur Diagnostik und Therapie zu verwenden. In meiner Praxis habe ich Erfahrungen mit dem System «SkaSys» (SkaSys Produktionsgesellschaft $\mathrm{mbH}$, Weiden i.d.Opf., Deutschland) gesammelt, mit dessen Hilfe man Belastungen und Therapiemittel anhand von biophysikalischen Reaktionen (Muskeltest, Pulsreaktionen usw.) ermitteln kann [23].

\section{Fallbeispiel}

Die besprochenen Theorien bieten aus der Sicht des Praktikers mögliche Erklärungen zu erstaunlichen Reaktionen, wie wir sie immer wieder beobachten können. Folgendes Beispiel eines 16-jährigen Mädchens mit starken Kopfschmerzen mag das veranschaulichen.

Aus dem Bericht der neurologischen Klinik des UniversitätsSpitals Zürich vom 4.5.2009:

Relevante Anamnese: Seit Oktober 2008, gleichzeitig mit der Entfernung einer Zahnspange, relativ rasch einsetzender, seither permanent vorhandener holokranieller, bifrontal betonter Kopfschmerz von hämmernd-pulsierendem Charakter und hoher Intensität (VAS 9-10!). Im Verlauf haben diese Kopfschmerzen tendenziell zugenommen. Die bisherige Therapie bestand aus NSAR, Defalgan, Aspirin und blieb bisher ohne günstige Auswirkung auf die Kopfschmerzen. Eine begleitende Photo- oder Phonophobie (bzw.) Nausea wird verneint. Durch die Kopfschmerzen ist die Konzentration eingeschränkt, in der Schule nimmt der Kopfschmerz weiter zu (von VAS 9 auf VAS 10 von 10). Seit Februar 2009 ist eine Teilnahme am Schulunterricht nur noch teilweise möglich, zwischen Februar und April 2009 war diese auf circa $3 \mathrm{~h}$ pro Woche beschränkt.

Eine fachärztliche neurologische Abklärung ergab im Januar 2009 einen unauffälligen neurologischen Status; ein MRI (Magnetic Resonance Imaging) des Neurocranium inkl. MRA und MR-Venogramm im März 2009 ergab einen Normalbefund. Eine Lumbalpunktion ergab eine Zellzahl/ $\mu$ l von 0 , ein normales Protein, negative OKB (oligoklonale Banden). Es wird eine antidepressive Therapie in Erwägung gezogen. Zwischenzeitlich erfolgte eine Behandlung mittels Bioresonanz und Akupunktur mit jeweils vorübergehender Besserung. Die Patientin besucht die Schule momentan circa $3 \mathrm{~h}$ pro Tag.

Familienanamnese ergab migräneartige Kopfschmerzen bei der Grossmutter mütterlicherseits.

Ausserdem erfolgte eine HPV(Human Papilloma Virus)-Impfung gegen Gebärmutterhalskarzinom im Oktober 2008, eine Appendektomie mit 4 Jahren und ein leichtes Anstrengungsasthma. Die Stimmung wird als fröhlich beschrieben, und sie hat weiterhin soziale Kontakte und treibt Sport. Noxen: Kein Nikotin, selten Äthyl. Weitere neurologische Untersuchungen ergaben keine relevanten pathologischen Befunde.

In der Beurteilung wird eine Diskrepanz zwischen der entspannt wirkenden und in ihrer Stimmung gut modulierenden Patientin mit erhaltenen sozialen und sportlichen Aktivitäten und der angegebenen stärksten Kopfschmerzen gemäss der visuell-analogen Schmerzskala erwähnt. Als wahrscheinlichste Diagnose wird chronischer Kopfschmerz vom Spannungstyp diagnostiziert. Der Therapievorschlag war schmerzdistanzierende Therapie, z.B. mit Duloxetin maximal 10 Tage pro Monat, sowie gegebenenfalls eine Verhaltenstherapie.

\section{Weiteres Vorgehen in der Praxis}

- 16.6.2009: Erstuntersuchung in meiner Praxis. Die Frage war, ob die Entfernung der Zahnspange eine Kaufunktionsstörung ausgelöst hatte, wie es der Akupunkteur aus Österreich vermutete. Ich konnte weder einen Zusammenhang mit dem Material noch mit der Funktion finden. - 18.6.2009: Mit dem System «SkaSys» [22, 23] testete ich folgende Belastungen: Viren und viroide Toxine als homöopathische Potenz D1, Impfnosode Tuberculinum bovinum D2 und den Impfzusatz Natrium Carbonicum D2, Prion C4 sowie eine Belastung der Niere als D3. Als Zielorgane gaben Dendriten, Mitochondrien, Ribosomen sowie der Golgi-Apparat Resonanz. Als Therapeutika ergaben sich Zincum valerianicum D200, Zinnober und Bachblüte Nr. 5. Die weitere Therapie bestand aus Bioresonanz mit den gefundenen Belastungen, Neuraltherapie sowie Scenar-Therapie, wobei mit kinesiologischen Techniken jeweils die Dosierung überprüft wurde. Nach drei weiteren Sitzungen (29.6., 3.7. und 8.7.) besserten sich die Kopfschmerzen etwas (VAS 7-8). - 28.8. und 4.9.2009: Weitere Therapie, diesmal zusätzlich mit dem HPVImpfstoff. Die subjektiven Beschwerden waren daraufhin verschwunden. Eine Nachtestung am 13.11. ergab immer noch eine Reaktion auf Tuberculinum bovinum und Prionen; eine Weiterbehandlung schien aber nicht nötig.

\section{Diskussion}

Die erwähnten Theorien deuten darauf hin, dass zur Erklärung der Phänomene lebender Systeme und Lebewesen nicht nur die Gesetze der klassischen Physik, sondern auch Phänomene der Quanten- und Chaostheorie herangezogen werden müssen. Deutlich gezeigt wurde das insbesondere im Zusammenhang mit einem pflanzlichen Subsystem, der Photosynthese [24].

Ein Hauptcharakteristikum dieser nichtklassischen Betrachtungen ist eine innere Dynamik der Materie, die durch Oszillationen charakterisiert werden kann. Moderne Biophysik betrachtet den lebenden Organismus daher mit seinen unzähligen rhythmischen Prozessen verschiedenster Frequenzen als ein komplexes, hochdynamisches und kohärentes System von oszillierenden Feldern, die über ihre Phasenbeziehungen nicht linear miteinander gekoppelt sind. Je harmonischer alle diese Prozesse miteinander ablaufen, desto stabiler hinsichtlich äusserer Reize und gesünder ist ein Organismus. Dabei spielen übergeordnete elektromagnetische Felder, und spekulativ 
auch Skalarwellen als ordnende Strukturen, eine entscheidende Rolle. Biochemische Prozesse werden durch diese Felder gesteuert und beeinflussen rückwirkend auch diese wieder. Dabei können Fremdstoffe diese Ordnungsstrukturen über Resonanzen auf vielfältige Weise beeinflussen.

Möchte man Phänomene wie Bewusstsein und Psyche, die res cogitans nach Descartes, auch in die Diskussion einbeziehen, was für den mit Patienten arbeitenden Praktiker unabdingbar ist, stellt sich die Frage, ob die Aufgabe überhaupt mit einer naturwissenschaftlichen Theorie lösbar ist. Dabei stimme ich Roger Penrose und dem Physiker Abner Shimony zu, die festhalten:

- Das Wesen des Geistes kann mit wissenschaftlichen Methoden behandelt werden.

- Die Konzepte der Quantenmechanik sind für das GeistKörper-Problem von Bedeutung.

- Es wird noch eine Modifikation des Quantenformalismus benötigt [11].

Ein Aspekt der Quantenmechanik ist der Doppelcharakter der Materie, die Dualität als Teilchen und Welle. Verschiedene Physiker erläuterten mir im Gespräch, Komplementärmedizin könnte sich eher auf die oszillierenden, dynamischen Eigenschaften der Materie beziehen, insbesondere unter Berücksichtigung von Resonanzphänomenen. Dank dieser Grundlagen werden auch Beobachtungen verständlich, die in der Komplementärmedizin als Störfeld bezeichnet werden. Ein in sich harmonisch schwingendes System wird durch ein anderes Feld überlagert und damit gestört [25]. Dabei können Symptome entstehen, die sich dem klassischen Krankheitsbegriff entziehen.

Einschränkend möchte ich festhalten, dass ich als medizinischer Praktiker die Grundlagen der erwähnten physikalischen Theorien nur im Ansatz erfassen kann. Ich beobachte aber individuelle Reaktionen bei meinen $\mathrm{Pa}-$ tienten und versuche diese mit den genannten Theorien zu erklären, wobei ich deren mathematische Struktur nicht vollständig zu kennen brauche. Es geht schlussendlich darum, die Möglichkeiten zu begreifen, die innerhalb eines Theorierahmens möglich sind.

Nach vielen Diskussionen mit verschiedensten Wissenschaftlern kann ich folgendes Erklärungsmuster erkennen: Je weniger diese die angesprochenen Grundlagen kennen, umso mehr sprechen sie bei für sie unerklärbaren Heilerfolgen von Placeboeffekten, ohne dies genauer erklären zu können. Beispielsweise gilt im Sinne des Reduktionismus allgemein, Homöopathie könne nicht wirken, weil kein Molekül im Wasser nachweisbar ist, während Quantenchemiker auf die faszinierenden Eigenschaften von Wasser hinweisen, wobei mir gegenüber einer einmal er- wähnte, wissenschaftlich spreche nichts gegen Homöopathie, auch wenn man den Wirkungsmechanismus noch nicht genau kenne.

Aus dieser Sicht muss man auch den besprochenen $\mathrm{Pa}-$ tientenfall diskutieren. Nach meiner Diagnose war es eine Impfkomplikation, wobei sich Bestandteile des Impfstoffes als Störfeld etablierten. Das kann, wenn wir die Erkenntnisse der Chaostheorie zu Hilfe nehmen, ein nicht reproduzierbares Einzelphänomen sein. Die Wechselwirkungen im Körper können über Biophotonen ablaufen. Besonders interessant ist dabei die Tatsache, dass die vom Testgerät «SkaSys» produzierten skalaren Wellen über kinesiologische Testungen zu dieser Diagnose führten. Die später durchgeführten Therapien erfolgten grösstenteils mit Informationen aus dem «SkaSys»-Testsystem. Unklar ist, welches System, etwa welche morphologischen Strukturen, als Empfänger solcher Informationstherapien wirkt. Doch da könnten die besprochenen Wasserstrukturen oder die Mikrotubuli eine wichtige Rolle spielen.

Aus Sicht der exakten Naturwissenschaften ist eine derartige Erklärung wohl ungenügend und sogar widersinnig. Hier setzt jedoch die anfangs angeführte Kritik an, nämlich dass mit ihren Regeln, wie der Forderung nach Reproduzierbarkeit, die Realität der Arzt-Patienten-Beziehung vermutlich nie voll erfasst werden kann. Das heisst nicht, dass diese Art zu forschen unsinnig wäre; wir verdanken der reduktionistischen Methodik sehr viel. Doch Erfolge in einem Bereich dürfen nicht zu einer Überinterpretation ihrer Aussagen führen.

Als Praktiker muss ich dem individuellen Einzelfall gerecht werden, in dem oft komplexe Zusammenhänge eine Rolle spielen. Derart komplexe Abhängigkeiten kann man mit den üblichen Doppelblindstudien, in denen man meistens nur zwei oder drei Faktoren in einen statistischen $\mathrm{Zu}$ sammenhang bringt, nicht erkennen. Als Beispiel mag die mögliche Interaktion von den 80 000-100 000 chemischen Verbindungen erwähnt sein, denen wir ausgesetzt sind. Das Beobachten und Beschreiben von auftretenden individuellen Phänomenen und das Suchen nach sinnvollen Erklärungen kann in Analogie mit dem geisteswissenschaftlichen Vorgehen gesetzt werden.

Ich bin heute überzeugt, dass wir komplementäre Verfahren aus dieser ganzheitlichen Sicht beurteilen müssen. Nach eigener Erfahrung bieten sie bei vielen Erkrankungen eine unverzichtbare Unterstützung [1].

\section{Disclosure Statement}

There is no conflict of interest to declare. 


\section{Literatur}

$>1$ Weilenmann U: Zahnärztliche Materialien. Eine kritische Wertung aus komplementärmedizinischer Sicht. Schweiz Monatsschr Zahnmed 2009;119:143-155.

-2 Weilenmann U: Ganzheitliche Zahnmedizin: Blosser Glauben oder wissenschaftliche Medizin? Schweiz Monatsschr Zahnmed 2000;110:269-281.

3 Störig HJ: Weltgeschichte der Philosophie. Zürich, Ex Libris, 1982.

4 Cramer F: Chaos und Ordnung. Die komplexe Struktur des Lebendigen, ed 2. Stuttgart, Deutsche Verlags-Anstalt, 1989.

5 Prigogine I: Die Gesetze des Chaos. Frankfurt, Insel, 1998.

6 Penrose R: Schatten des Geistes. Weg zu einer neuen Physik des Bewusstseins. Heidelberg, Spektrum, 1995.

7 Görnitz T: Quanten sind anders. Die verborgene Einheit der Welt. Heidelberg, Spektrum, 1999.

8 Fröhlich H: Biological Coherence and Response to External Stimuli. Berlin, Springer, 1988

9 Hyland GJ: Fröhlich's physical theory of cancer - Fröhlich's path from theoretical physics to biology, and the cancer problem. Neural Netw World 2009;19:337-354.

10 Popp FA: Neue Horizonte in der Medizin, ed 3. Stuttgart, Haug, 2006.
11 Penrose R: Das Grosse, das Kleine und der menschliche Geist. Heidelberg, Spektrum, 1998.

12 Pokorný J: Fröhlich's coherent vibrations in healthy and cancer cells. Neural Netw World 2009;19:369-378.

13 Trincher K: Wasser. Grundstruktur des Lebens und Denkens. Wien, Herder, 1990.

14 Del Giudice E, Elia V, Tedeschi A: Role of water in the living organisms. Neural Netw World 2009;19:355-360.

15 Voeikov V, Del Giudice E: Water respiration the basis of the living state. Water 2009;1:5275.

16 Voeikov V: Fundamental role of water in bioenergetics; in Belousov L, Voeikov V, Martynyuk V: Biophotonics and Coherent Systems in Biology. New York, Springer, 2007, pp 89-104.

17 Internationales Institut für Biophysik: Informationen zur Biophotonenforschung. www.biophotonik.de

18 Galle M: Untersuchungen zum dichte- und zeitabhängigen Verhalten der ultraschwachen Photonenemission von pathogenetischen Weibchen des Wasserflohs Daphnia magna. Inaugural-Dissertation. Saarbrücken, Universität Saarbrücken, 1993.
19 Popp FA: Essential differences between coherent and non-coherent effects of photon emission from living organisms; in Sheng X, Van Wijk R (eds): Biophotonics: Optical Science and Engineering for the 21st Century. New York, Springer, 2005, pp 109-124.

20 Popp FA, Chang JJ, Herzog A, Yan Z, Yan Y: Evidence of non-classical (squeezed) light in biological systems. Phys Lett A 2002;293:98102.

21 Meyl K: Neutrinopower. Johannes von Butlar im Gespräch mit Prof. Konstantin Meyl. Marktoberdorf, Argo, 2000

22 Bischof M: Tachyonen, Orgonenergie, Skalarwellen. Feinstoffliche Felder zwischen Mythos und Wissenschaft. Aarau, AT, 2002.

23 Lechner J: Störfelddiagnostik, Medikamenten- und Materialtest. Kötzting, Verlag für Ganzheitliche Medizin, 2000.

24 Collini E, Wong CY, Wilk KE, Curmi PM, Brumer P, Scholes GD: Coherently wired light-harvesting in photosynthetic marine algae at ambient temperature. Nature 2010; 463:644-647.

25 Graf K: Ganzheitliche Zahnmedizin. Fakten, Wissenswertes, Zusammenhänge. Stuttgart, Sonntag, 2000, p 54. 